\title{
BMJ Open Summarising and synthesising regression coefficients through systematic review and meta-analysis for improving hypertension prediction using metamodelling: protocol
}

To cite: Chowdhury MZI, Naeem I, Quan $\mathrm{H}$, et al. Summarising and synthesising regression coefficients through systematic review and meta-analysis for improving hypertension prediction using metamodelling: protocol. BMJ Open 2020;10:e036388. doi:10.1136/ bmjopen-2019-036388

- Prepublication history for this paper is available online To view these files, please visit the journal online (http://dx.doi. org/10.1136/bmjopen-2019036388).

Received 12 December 2019 Revised 05 March 2020 Accepted 20 March 2020

Check for updates

(C) Author(s) (or their employer(s)) 2020. Re-use permitted under CC BY-NC. No commercial re-use. See rights and permissions. Published by BMJ.

For numbered affiliations see end of article.

Correspondence to Mohammad Ziaul Islam Chowdhury; mohammad.chowdhury@ ucalgary.ca

\section{ABSTRACT}

Introduction Hypertension is one of the most common medical conditions and represents a major risk factor for heart attack, stroke, kidney disease and mortality. The risk of progression to hypertension depends on several factors, and combining these risk factors into a multivariable model for risk stratification would help to identify high-risk individuals who should be targeted for healthy behavioural changes and/or medical treatment to prevent the development of hypertension. The risk prediction models can be further improved in terms of accuracy by using a metamodel updating technique where existing hypertension prediction models can be updated by combining information available in existing models with new data. A systematic review and meta-analysis will be performed of hypertension prediction models in order to identify known risk factors for high blood pressure and to summarise the magnitude of their association with hypertension.

Methods and analysis MEDLINE, Embase, Web of Science, Scopus and grey literature will be systematically searched for studies predicting the risk of hypertension among the general population. The search will be based on two key concepts: hypertension and risk prediction. The summary statistics from the individual studies will be the regression coefficients of the hypertension risk prediction models, and random-effect meta-analysis will be used to obtain pooled estimates. Heterogeneity and publication bias will be assessed, along with study quality, which will be assessed using the Prediction Model Risk of Bias Assessment Tool checklist.

Ethics and dissemination Ethics approval is not required for this systematic review and meta-analysis. We plan to disseminate the results of our review through journal publications and presentations at applicable platforms.

\section{INTRODUCTION}

Hypertension or high blood pressure is one of the most common medical conditions affecting almost one in four individuals worldwide ${ }^{1}$ and represents a major risk factor for heart attack, stroke, kidney disease and
Strengths and limitations of this study

- One of the big strengths of this study is the comprehensiveness of the systematic review, which will include a search of four different databases and extensive use of reference lists.

- This study will provide a comprehensive summary of hypertension risk prediction models and the risk factors they employ in the general population, and will summarise evidence as to their performance.

- The study will lay a foundation for further improvement on hypertension risk prediction using metamodelling, where pooled common regression coefficients obtained through meta-analysis will be used in a new population.

- This review has some limitations due to inevitable heterogeneity caused by study differences in modelling methods, adjustment factors and methods of measurements.

mortality. ${ }^{2}$ Hypertension has been attributed to be responsible for $13 \%$ of global deaths. ${ }^{3}$ Prevention of hypertension, as well as control of blood pressure in hypertensive patients, is considered a major public health and primary care concern. ${ }^{4}$ Current population health research is increasingly integrating aspects of precision public health, which emphasises targeted health interventions by identifying people who are at greatest risk of disease. ${ }^{5}$

The risk of developing hypertension depends on many factors, including age, body mass index (BMI), blood pressure, smoking, family history and level of physical inactivity. ${ }^{6}$ Combining these known risk factors into a multivariable model for risk stratification would help to identify highrisk individuals who should be targeted for healthy behavioural changes and/or medical treatment to prevent the development of 
hypertension. $^{78}$ Inaccurate risk estimation can lead to ineffective interventions in patients at low risk, as well as missed opportunities to intervene in those who are most susceptible to develop hypertension.

The accuracy of the prediction model can be improved by using a metamodel updating technique. ${ }^{9}$ Existing hypertension prediction models can be updated by combining information from other published models. This metamodel updating approach works from the 'middle ground' in which existing prediction models designed for the specific populations and endpoints of interest are then used and revised to fit a new population. ${ }^{910}$ The updated model is then based on both the new and existing data, with the potential of improving model performance in the new population of interest.

Many prediction models have been developed to predict the risk of hypertension in the general population. Although there are multiple models, their predictive ability varies due to a lack of consistency in estimating risk by different models. Two prior reviews examined hypertension risk prediction models in adults. ${ }^{1112}$ Both reviews conducted a narrative synthesis of the evidence to summarise the existing knowledge and performance of hypertension prediction models. In addition, a systematic review on prediction models to identify children at increased risk of future hypertension was also conducted. ${ }^{13}$ However, none of the previous studies performed a metaanalysis. Our review is different from the past reviews and adds to knowledge on predicting risk of hypertension and identifying associated risk factors in the following ways: (1) we plan to synthesise common regression coefficients of the existing hypertension risk prediction models through meta-analysis; (2) we propose pooled regression coefficients to be further used in developing a new hypertension risk prediction model for improved accuracy; (3) we plan to provide a detailed assessment of quality of studies among models developed; and (4) we hope to identify several additional and recently derived models. A systematic review and meta-analysis will be performed to identify existing prediction models for incident hypertension and risk factors they employ in the general adult population and to summarise evidence as to their performance. This will allow for model updating, which has the potential of improving the predictive ability for hypertension.

\section{Objectives}

The specific objectives for this systematic review and meta-analysis are

1. To summarise the predictive ability and quality of all existing risk prediction models for incident hypertension.

2. To identify a list of risk factors/predictors considered in existing hypertension risk prediction models and get their corresponding parameter values (regression coefficients).

3. To lay a foundation for further improvement on hypertension risk prediction through metamodel updating technique using pooled common regression coefficients identified in objective 2 .

\section{METHOD}

\section{Protocol design}

A systematic review and meta-analysis study design will be used to summarise information about all known published hypertension risk prediction models. To summarise and synthesise research evidence from multiple studies that are combinable, this study design is most applicable. This study protocol is reported in accordance with the Preferred Reporting Items for Systematic Reviews and Meta-Analyses Protocol. ${ }^{14}$

\section{Data sources and searches}

We will systematically search MEDLINE, Embase, Web of Science and Scopus (each from inception to March 2020) for studies predicting the risk of incident hypertension in the general adult population. We will also search the reference lists of all identified relevant articles and will contact experts in the field of hypertension for information about other potential ongoing or unpublished studies. These experts will be identified from the review process. For the grey literature, Google Scholar and ProQuest (theses and dissertations) will be searched. The systematic search will be broken into two key concepts: hypertension and risk prediction. For each key concept, proper free-text words and Medical Subject Headings (MeSH) terms will be used. In addition, certain text words will be truncated, or wildcards will be used to capture all relevant articles from all databases. Finally, all the words and MeSH terms will be combined using the Boolean operators 'AND', 'OR' and 'NOT' as appropriate. The detailed search strategy for MEDLINE is provided in box 1 .

\section{Eligibility criteria}

We plan to use broad inclusion criteria to provide a comprehensive systematic review of the topic. Only primary studies with extractable data will be included in this review. This excludes reviews, editorials, commentaries and letters to the editor. We will only consider cohort studies considering the risk prediction models for incident hypertensions are generally developed using cohort study design with a follow-up information. However, studies will not be considered for inclusion if they were written in languages other than English and French. The Population, Prognostic Factors (or Models of Interest) and Outcome ${ }^{15}$ framework will used to outline eligibility criteria.

\section{Population}

The study population consists of general people who are free from hypertension at baseline and those for which hypertension risk prediction models are developed. There will be no restrictions in geographical region, time period or gender of the study participants. However, only the adult population, those who are aged 18 years or older, will be considered as our outcome is essential hypertension common in adults.

Prognostic Factors (or Models of Interest)

Studies that developed risk prediction models for incidence hypertension in the general adult population 
Box 1 Keywords used to search in MEDLINE

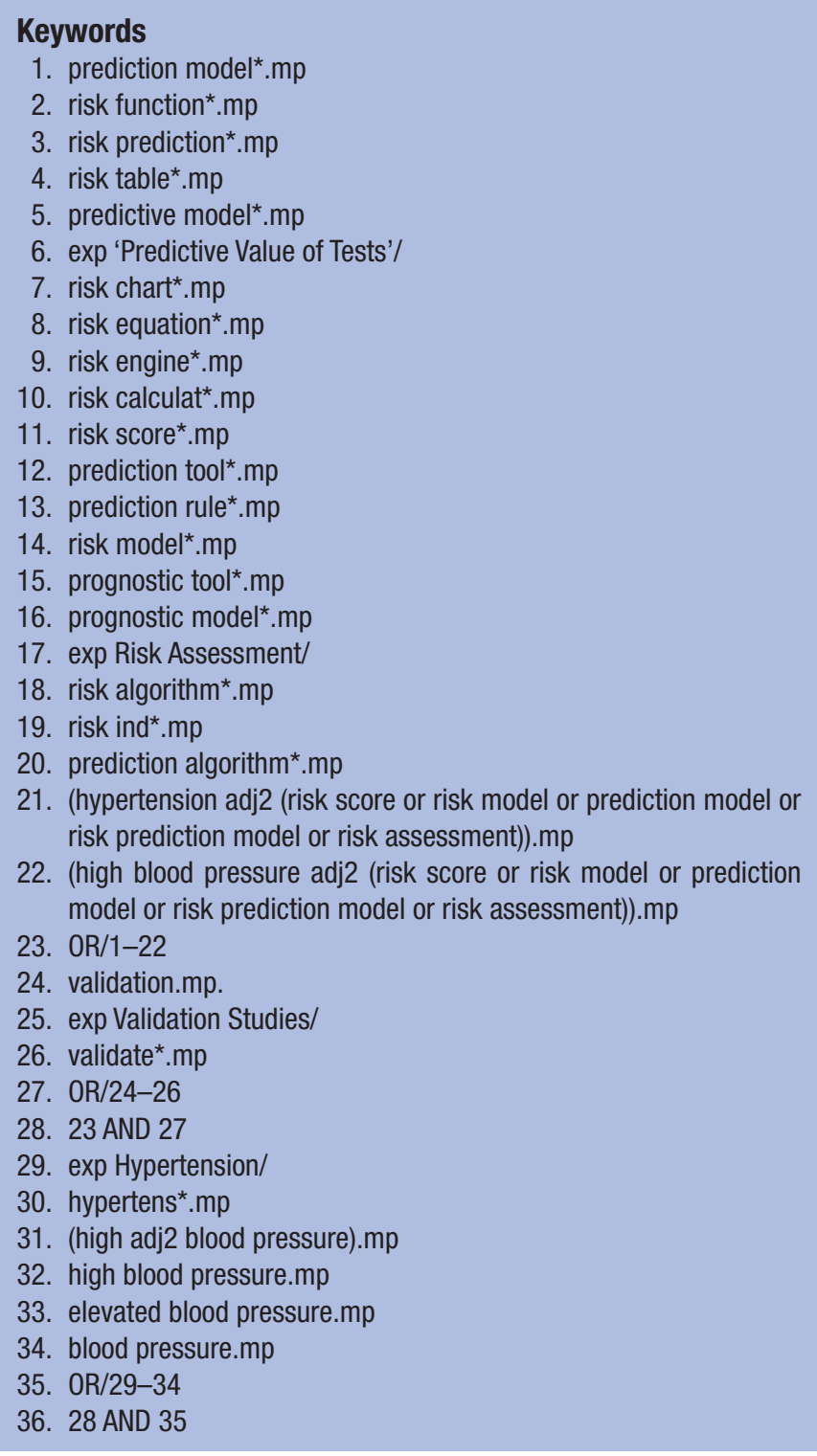

will be included. However, studies focusing on the additive predictive value of new risk factors to an existing prediction model, or studies presenting a prediction model developed in patients with previous hypertension, or studies that derived risk prediction tools other than score-type tools, such as risk charts, will not be included. In addition, we will not consider studies that have only assessed bivariate associations between predictors and hypertension incidence. Rather, we will be focusing on those studies where hypertension prediction models were built incorporating predictors that demonstrated significant prognostic contribution in predicting incident hypertension. Further, we will only consider predictors that are non-invasive, considering their feasibility in routine setting. A hypertension risk prediction model is defined as one combining two or more predictors to obtain estimates of the predicted risk of developing hypertension. Regression coefficients associated with the risk prediction models are constants (that are estimated) in the prediction model that tells about the change in outcome corresponding to the change in predictors and describe the relationship between a predictor and the outcome. If a model is assessed on more than one external population, information from all reported models will be considered. However, if the model is presented both in a derivation cohort and in a validation cohort, only information from the validation cohort will be considered.

\section{Outcome}

Outcomes of our study are hypertension and will be primarily defined as a systolic blood pressure (SBP) of $\geq 140 \mathrm{~mm} \mathrm{Hg}$ or a diastolic blood pressure (DBP) of $\geq 90 \mathrm{~mm}$ $\mathrm{Hg}$ or taking antihypertensive medication. Although the 2017 American College of Cardiology (ACC)/American Heart Association (AHA) Hypertension Guideline lowers the cut-off level to define hypertension ( $\mathrm{SBP} \geq 130 \mathrm{~mm} \mathrm{Hg}$ or $\mathrm{DBP} \geq 80 \mathrm{~mm} \mathrm{Hg}$ or taking antihypertensive medication), ${ }^{16}$ we will consider the former definition bearing in mind prediction models developed before appearance of new definition considered the former definition. Nevertheless, if we identify any study that reported a different formal definition of hypertension (eg, new ACC/AHA definition), we will also consider those definitions to capture the maximum number of studies. In the event of more than one hypertension definition, meta-analysis will be performed separately for each different definition of hypertension (outcome).

\section{Study selection}

\section{Title-abstract screening}

Initially, all articles identified through our search strategy will be exported to EndNote (Clarivate Analytics) (a software for managing bibliographies, citations and references) for duplicate removal. Level 1 screening will consist of an independent review by two researchers of the title and abstract of deduplicated records. Studies will be included if they indicate assessment of prediction models for hypertension. Records will be included if they discuss either validation or development of such a model. Further, records will be included if they assess the predictive ability of individual risk factors associated with hypertension. Inter-rater reliability (kappa coefficient) will be calculated to measure disagreement between independent reviewers, which will be resolved by consensus. Studies kept during level 1 screening will go through level 2 screening.

\section{Full-text screening}

Full text articles will be retrieved for all records meeting level 1 review criteria. Level 2 screening will consist of full-text review by two independent reviewers. Articles will be included if they contain extractable data on hypertension prediction models and regression coefficients for hypertension risk factors. The articles identified eligible after full-text review will be included for data extraction. Agreement between reviewers will be quantified using the 
Records identified through database searching $(\mathrm{n}=)$

MEDLINE: , EMBASE: , Web of Science: , Scopus:
Additional records identified through other sources

$(\mathrm{n}=)$

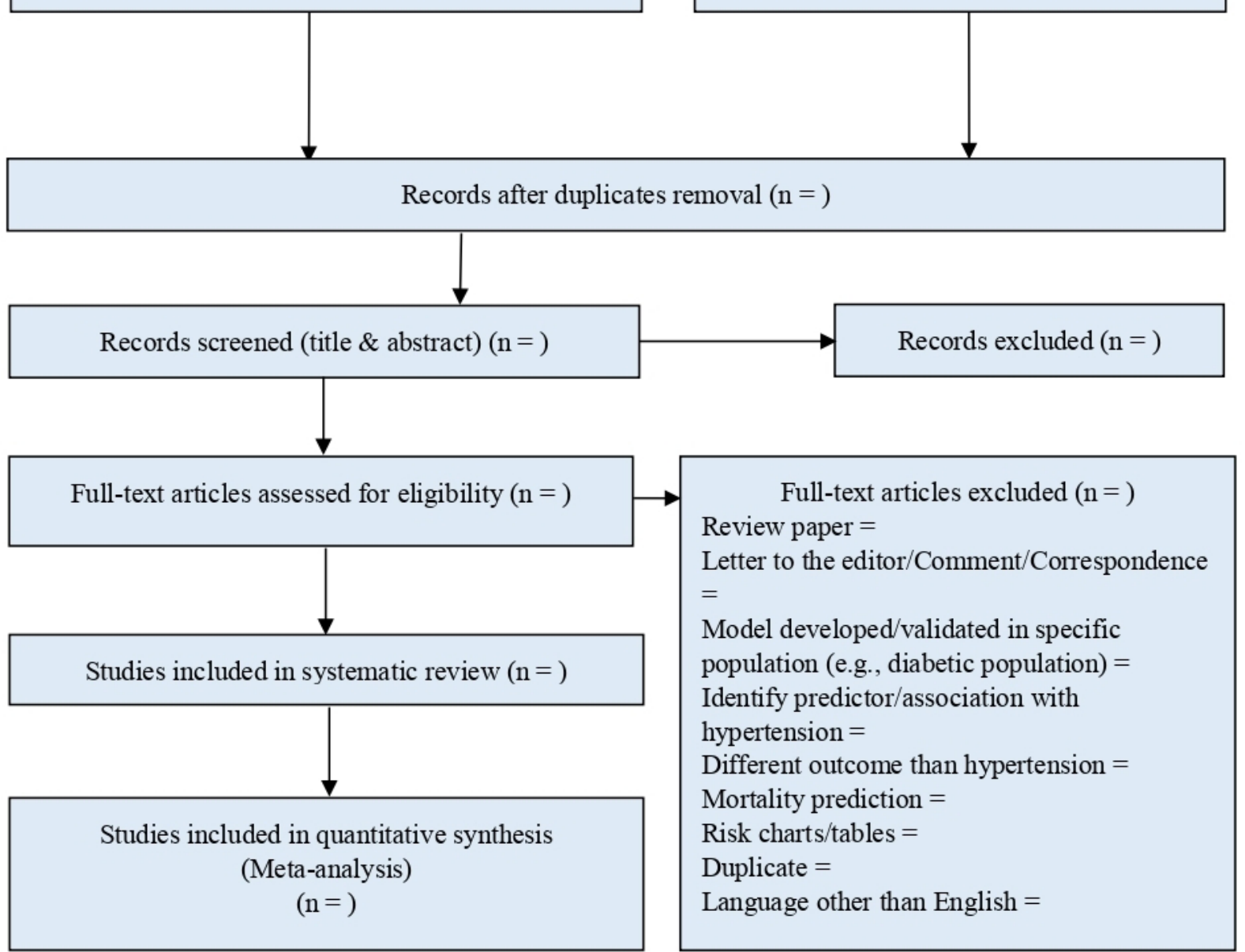

Figure 1 Preferred Reporting Items for Systematic Reviews and Meta-Analyses diagram for systematic review of studies presenting hypertension prediction models developed in the general population.

kappa statistic. Any disagreement between reviewers will be resolved through consensus.

\section{Data extraction and synthesis}

First, the numbers of studies identified, excluded (with the reason for exclusion) and included in the systematic review and subsequent meta-analysis will be summarised using the Preferred Reporting Items for Systematic Reviews and Meta-Analyses flow diagram ${ }^{17}$ (figure 1 ). Next, two reviewers will independently extract data from each study using a standardised form (table 1) that will include study name, location where the model was developed and participants' ethnicity, study design used, age and gender of the study participants, predictors included in the model, number of events and total participants, definition used for hypertension, duration of follow-up, modelling method used, measures of discrimination and calibration of the prediction model, and the validation of the prediction model. In a separate form (table 2), information about the parameters (regression coefficients) of the identified hypertension risk prediction models will be extracted, which includes study name, sample size, modelling method, mathematical model and corresponding regression coefficients, number of predictors, list of predictors, values of the regression coefficients and statistical significance of the regression coefficients. In this review, we will primarily focus on a traditional regression type's prediction modelling (eg, logistic regression model and Cox proportional hazard regression model), anticipating that it is the most common form of prediction modelling. Recently emerged more complicated modelling strategies (eg, machine learning tools) will not be considered in this review. It is very likely that some of the studies will report ORs or HRs (depending on the modelling methods) instead of the regression coefficients for their model. In that case, we will back-calculate the regression coefficients from the OR or HR using appropriate formula. For example, the OR formula for any single generally defined exposure variable $E$, whether 
dichotomous, ordinal or interval, controlling for a collection of control variables is given by

$$
\mathrm{OR}_{\mathrm{E}^{\mathrm{a}} \text { vs } \mathrm{E}^{\mathrm{b}}}=\exp \left[\left(\mathrm{E}^{\mathrm{a}}-\mathrm{E}^{\mathrm{b}}\right) \beta+\left(\mathrm{E}^{\mathrm{a}}-\mathrm{E}^{\mathrm{b}}\right) \sum_{\mathrm{j}=1}^{\mathrm{p}} \delta_{\mathrm{j}} \mathrm{W}_{\mathrm{j}}\right]
$$

where $E^{a}$ and $E^{b}$ are the two levels of the exposure variable $E, \beta$ is the regression coefficient associated with exposure $E$, and the deltas $(\delta s)$ are coefficients of potential interaction effects involving $E$ separately with each of the $p$ potential effect modifiers $W s{ }^{18}$ When there is no interaction case, the OR formula reduces to the quantity $\exp \left[\left(E^{a}-E^{b}\right) \beta\right]$ and becomes exp $(\beta)$, when the difference between exposure levels equals 1 . This formula can be used to convert ORs to the regression coefficients. Similarly, the HRs can also be converted to the regression coefficients. As relation between OR and HR is not yet established, pooling regression coefficients derived from them will be separate.

Meta-analysis of regression coefficients associated with the risk factors in prediction models involved several challenges, including incomparable effect measures (eg, OR and HRs); different methods of measurement for predictors and outcome; different approaches to handling outcome and predictors (eg, age can be continuous or categorical with different levels of categories and cut-offs to define categories); differences in measurement units; lack of proper reporting of estimates (eg, SE of the effect measures may not be reported); and use of a different set of adjustment predictors in different models. ${ }^{19}$ These issues can lead to a substantial amount of heterogeneity.

A recent guideline has provided some recommendations to overcome these issues. ${ }^{19}$ A separate meta-analysis on similar effect measures (eg, meta-analysis of ORs and HRs separately), similar associations (eg, meta-analysis of unadjusted and adjusted predictors separately), similar cut-offs for predictors (eg, separate meta-analysis on distinct cut-offs of predictors), and similar method of measurement for predictors and outcome (eg, separate meta-analysis for each measurement method) are among the recommendations.

Although ideal but very unlikely, the estimates (eg, regression coefficients or ORs or HRs) derived from different models are adjusted for the same set of other predictors. Estimates adjusted for a different set of predictors create difficulty in interpreting meta-analysis results. To overcome this issue, the guideline recommended considering meta-analysis only on those estimates that are adjusted for at least a predefined minimum core set of established predictors. ${ }^{19}$ This core set of predictors for the outcome can be defined in consultation with the experts. For the hypertension outcome, a potential core set of predictors to adjust can be age, gender, smoking status and BMI.

Standardisation of the effect measures to ensure they all relate to the same scale of measurement will also be considered if necessary. Regression coefficients will be converted into standardised regression coefficients and 
Table 2 Extracted information about the parameters of the existing hypertension prediction models

\begin{tabular}{|c|c|c|c|c|c|c|c|}
\hline Study & $\begin{array}{l}\text { Sample size } \\
\text { considered } \\
\text { in the final } \\
\text { model }\end{array}$ & $\begin{array}{l}\text { Modelling } \\
\text { method } \\
\text { used to } \\
\text { develop the } \\
\text { model }\end{array}$ & $\begin{array}{l}\text { Reported } \\
\text { mathematical } \\
\text { model and } \\
\text { corresponding } \\
\text { regression } \\
\text { coefficients of } \\
\text { the model }\end{array}$ & $\begin{array}{l}\text { Predictors } \\
\text { considered } \\
\text { in the final } \\
\text { model (n) }\end{array}$ & $\begin{array}{l}\text { List of } \\
\text { predictors } \\
\text { considered } \\
\text { in the final } \\
\text { model }\end{array}$ & $\begin{array}{l}\text { Values of } \\
\text { the reported } \\
\text { regression } \\
\text { coefficients/ } \\
\text { ORs in the } \\
\text { final model }\end{array}$ & $\begin{array}{l}\text { Statistical } \\
\text { significance } \\
\text { of the } \\
\text { corresponding } \\
\text { regression } \\
\text { coefficients }\end{array}$ \\
\hline
\end{tabular}

Reported mathematica

Sample size onsidered in the final develop the then meta-analysis will be performed. Most of the existing standardisation techniques ${ }^{2021}$ were built with continuous response variables in mind, and complication arises when response variable become binary as in our case. We will adopt the latent-theoretical method proposed by Grace et $a l^{22}$ for standardisation as this method offers a suitable approach for standardisation in a binary response variable.

We will follow the above steps and recommendations provided by the guideline ${ }^{19}$ in our study to make our meta-analysis results (pooled estimates) more interpretable. Although our initiatives may not provide complete remedy and heterogeneity may remain, we anticipate performing a random-effect meta-analysis will allow for inevitable heterogeneity in effect measures across studies.

\section{Quality assessment}

Study quality will be assessed by each reviewer, according to the Prediction Model Risk of Bias Assessment Tool (PROBAST) checklist $^{23}$ (table 3). The PROBAST tool is designed for assessing the risk of bias and concerns regarding the applicability of both diagnostic and prognostic prediction model studies. The PROBAST contains 20 questions under four domains: participants, predictors, outcome and analysis that facilitates judgement of risk of bias and applicability. Overall risk of bias of the prediction models will be judged as 'low', 'high' or 'unclear', and overall applicability of the prediction models will be judged as 'low concern', 'high concern' and unclear according to the PROBAST checklist.

\section{Data analysis}

The summary statistics, also known as the effect measure, from the individual studies will be the regression coefficients of the hypertension risk prediction models. Studies will be grouped based on the type of modelling method used to develop the model as regression coefficients will have different meanings based on different modelling methods (eg, in a logistic regression model, a coefficient represents the change in the log odds that would result from a one unit change in one specific predictor, holding all other predictors constant while in a Cox proportional hazard regression model, the coefficients represent the change in the expected log of the HR relative to a one unit change in one specific predictor, holding all other predictors constant). We will use random-effect metaanalysis to obtain the pooled weighted average of the common regression coefficients with $95 \%$ CIs for groups of models using the DerSimonian and Laird method. ${ }^{24}$ Forest plots will be also generated to show the pooled regression coefficient, together with $95 \%$ CI, the author's name, publication year and study weights. In studies that provided only regression coefficients $(\beta)$ but no measure of its variance or CIs, the SE and $95 \%$ CI of the regression coefficients $(\beta)$ will be calculated using the formula

$\operatorname{SE}(\beta)=\sqrt{\frac{\beta[1-\beta]+\left[N_{1}-1\right] \times\left[\beta /(2-\beta)-\beta^{2}\right]+\left[N_{2}-1\right] \times\left[2 \beta^{2} /(1+\beta)-\beta^{2}\right.}{\left(N_{1} \times N_{2}\right)}}$

where $N_{1}=$ the number of patients with hypertension and $N_{2}=$ the number of patients without hypertension and the upper $95 \% \mathrm{CI}=\beta+[1.96 \times \operatorname{SE}(\beta)]$, and lower $95 \% \mathrm{CI}=\beta-[1.96 \times \operatorname{SE}(\beta)]{ }^{25}$ If the CIs of the regression coefficients are available, SEs of the regression coefficients will be derived from the CIs. Information about the pooled regression coefficients will be presented in a tabular form (table 4). STATA V.13.1 will be used to perform statistical analysis using the following commands: metan, metareg, metabias, metatrim and metafunnel.

\section{Testing for heterogeneity}

Studies that will be included in our systematic review are likely to be diverse methodologically, and as a result, heterogeneity in the results is to be expected. Heterogeneity arises when differences between study results are 
Table 3 Prediction Model Risk of Bias Assessment Tool checklist

\begin{tabular}{|c|c|c|c|}
\hline Participants & Predictors & Outcome & Analysis \\
\hline $\begin{array}{l}\text { 1. Were appropriate data } \\
\text { sources used, for example, } \\
\text { cohort, RCT or nested case- } \\
\text { control study data? }\end{array}$ & $\begin{array}{l}\text { 1. Were predictors defined } \\
\text { and assessed in a similar way } \\
\text { for all participants? }\end{array}$ & $\begin{array}{l}\text { 1. Was the outcome } \\
\text { determined appropriately? }\end{array}$ & $\begin{array}{l}\text { 1. Were there a reasonable } \\
\text { number of participants with the } \\
\text { outcome? }\end{array}$ \\
\hline
\end{tabular}

2. Were all inclusions and exclusions of participants appropriate?

$\begin{array}{ll}\text { appropriate? } & \text { knowledge of outcome data? } \\ \text { at the time the model is } \\ \text { intended to be used? }\end{array}$

2. Was a prespecified or standard outcome definition used?

3. Were predictors excluded from the outcome definition?

4. Was the outcome defined and determined in a similar way missing data handled for all participants?

5. Was the outcome determined without knowledge of predictor information?

6. Was the time interval between predictor assessment and outcome determination appropriate? appropriately? appropriately? avoided?

\section{Were continuous and} categorical predictors handled

3. Were all enrolled participants included in the analysis?

4. Were participants with

5. Was selection of predictors based on univariable analysis

6. Were complexities in the data (eg, censoring, competing risks and sampling of control participants) accounted for appropriately?

7. Were relevant model performance measures evaluated appropriately?

8. Were model overfitting, underfitting and optimism in model performance accounted for?

9. Do predictors and their assigned weights in the final model correspond to the results from the reported multivariable analysis?

$\mathrm{RCT}$, randomised controlled trial.

Table 4 Information about the pooled regression coefficients

\begin{tabular}{|c|c|c|c|c|c|}
\hline $\begin{array}{l}\text { lame of the predictor } \\
\text { xtracted from the } \\
\text { elected model }\end{array}$ & $\begin{array}{l}\text { Names of } \\
\text { the studies } \\
\text { from which } \\
\text { predictor was } \\
\text { extracted }\end{array}$ & $\begin{array}{l}\text { Studies reporting } \\
\text { the regression } \\
\text { coefficient of the } \\
\text { corresponding } \\
\text { predictor (n) }\end{array}$ & $\begin{array}{l}\text { Reported values } \\
\text { of regression } \\
\text { coefficients from } \\
\text { the corresponding } \\
\text { predictor }\end{array}$ & $\begin{array}{l}\text { Pooled value of } \\
\text { the corresponding } \\
\text { regression } \\
\text { coefficients with } \\
95 \% \text { Cls }\end{array}$ & $\begin{array}{l}\text { Amount of } \\
\text { heterogeneity } \\
\text { observed }\end{array}$ \\
\hline
\end{tabular}
selected model

95\% Cls 
beyond those attributable to chance alone; potential sources include the study setting, the study participant type and the study methodology, among others. Heterogeneity will be assessed using Cochran's $Q$ statistic and quantified with the $\mathrm{I}^{2}$ statistic. A p value of less than 0.05 will be considered statistically significant heterogeneity and will be categorised as low, moderate and high when the $\mathrm{I}^{2}$ values are below $25 \%$, between $25 \%$ and $75 \%$, and above $75 \%$, respectively. ${ }^{26}$ Sources of heterogeneity will be further explored using metaregression and stratified analyses according to the modelling method and study characteristics (sample size, duration of follow-up, number of predictors included within the prediction models and geographical location).

\section{Assessment of publication bias}

Publication bias, a tendency from authors to publish favourable or significant results, threatens the validity of a review's findings. Synthesised results through metaanalysis from the systematic review can produce overstated conclusions due to publication bias. ${ }^{27}$ Publication bias will be examined using a funnel plot and Egger's test. Funnel plots provide visual aid for detecting publication bias in systematic reviews. A symmetrical inverted funnel plot will indicate absence of publication bias, whereas an asymmetrical funnel plot will indicate presence of publication bias. ${ }^{28}$ We will apply the funnel plot to visually describe the publication bias. Egger's test will be performed to formally test/confirm the existence of publication bias. ${ }^{28}$ If publication bias is identified, we will apply Duval and Tweedie's trim and fill method as a form of sensitivity analysis to see how bias in the pooled estimates can potentially be reduced. ${ }^{29}$

\section{Patient and public involvement}

Patients and the public will not be involved in the development, design, conduct or reporting of the study.

\section{Ethics and dissemination}

There is no involvement of human subjects in this study as there will be no primary data collection. So ethical approval is not required for this systematic review. A manuscript will be prepared for peer-reviewed journals after completion of the full analysis. Also, findings will be presented at relevant conferences and seminars.

\section{DISCUSSION}

This systematic review will provide information on available hypertension risk prediction models, their performances and their study quality. Additionally, it will provide information on risk factors/predictors that were considered during the model development and their corresponding parameter values (regression coefficients). Together, this information will deliver a comprehensive summary of the predictive ability of these models based on the reported data. Previous hypertension risk prediction models consider various demographic and clinical characteristics, and blood biomarkers, including SBP, paternal history of hypertension, BMI, age and sex. ${ }^{30} 31$ The Framingham risk score is one of the most widely used for hypertension risk prediction modelling and risk assessment. ${ }^{32}$ However, it is imperative to regularly review and synthesise risk prediction models for hypertension as research in risk factors of hypertension improve and data for further clinical and demographic biomarkers become available. Using methodologies to evaluate prediction model performance and accuracy, we found that the metamodelling technique allows for improvement on existing hypertension prediction models. This compilation of both existing and new information allows for an effective technique to improve precision public health in the corresponding populations. ${ }^{33} 34$

This systematic review has its strengths in an effective and systematic search strategy. With a broad inclusion criterion, we hope to obtain all relevant literature on hypertension risk models. Further, the unique pooled analysis of regression coefficient provides an opportunity to synthesise large amounts of information's on risk factors for hypertension. This review also has some limitations. Although the English language is generally perceived to be the universal language of science, selection of research findings in a particular language (eg, English and French) can introduce language bias and may lead to erroneous conclusions. With this in mind, readers should be cautious when interpreting the findings of our results. Pooling regression coefficients through meta-analysis is also challenging, considering inevitable heterogeneity caused by study differences in modelling methods, adjustment factors and methods of measurements. We urge readers to be cautious while interpreting the findings of the summary results.

In conclusion, this systematic review has the potential to synthesise the overall evidence on the performance and effectiveness of hypertension prediction models and summarise the current development status. This will provide updated tools for healthcare providers and clinical practice, along with advancing the field of hypertension research.

\section{Author affiliations}

${ }^{1}$ Department of Community Health Sciences, University of Calgary Cumming School of Medicine, Calgary, Alberta, Canada

${ }^{2}$ Department of Medicine, University of Calgary Cumming School of Medicine, Calgary, Alberta, Canada

${ }^{3}$ Health Status Assessment, Surveillance and Reporting, Public Health Surveillance and Infrastructure, Population, Public and Indigenous Health, Alberta Health Services, Calgary, Alberta, Canada

${ }^{4}$ Department of Family Medicine, University of Calgary Cumming School of Medicine, Calgary, Alberta, Canada

Contributors All authors contributed to this work. MZIC and TCT contributed to the conception and design of the review. MZIC and IN read and screened abstracts and titles of potentially relevant studies. MZIC and IN read the retained papers and were responsible for extracting data and rating their quality independently. MZIC drafted the paper and IN, HQ, AAL, KCS, MOB and TCT critically reviewed it and suggested amendments prior to submission. All authors approved the final version of the manuscript and take responsibility for the integrity of the reported findings. 
Funding The authors have not declared a specific grant for this research from any funding agency in the public, commercial or not-for-profit sectors.

Competing interests None declared.

Patient consent for publication Not required.

Provenance and peer review Not commissioned; externally peer reviewed.

Open access This is an open access article distributed in accordance with the Creative Commons Attribution Non Commercial (CC BY-NC 4.0) license, which permits others to distribute, remix, adapt, build upon this work non-commercially, and license their derivative works on different terms, provided the original work is properly cited, appropriate credit is given, any changes made indicated, and the use is non-commercial. See: http://creativecommons.org/licenses/by-nc/4.0/.

ORCID iDs

Mohammad Ziaul Islam Chowdhury http://orcid.org/0000-0002-5397-2773

Tanvir C Turin http://orcid.org/0000-0002-7499-5050

\section{REFERENCES}

1 Mills KT, Bundy JD, Kelly TN, et al. Global disparities of hypertension prevalence and control: a systematic analysis of population-based studies from 90 countries. Circulation 2016;134:441-50.

2 Centers for Disease Control and Prevention. High blood pressure. Available: https://www.cdc.gov/bloodpressure/effects.htm

3 World Health Organization, Mendis S, Puska P, Norrving B, eds. Global atlas on cardiovascular disease prevention and control. Geneva: World Health Organization, 2011.

4 Otsuka T, Kachi Y, Takada H, et al. Development of a risk prediction model for incident hypertension in a working-age Japanese male population. Hypertens Res 2015;38:419-25.

5 Khoury MJ, lademarco MF, Riley WT. Precision public health for the era of precision medicine. Am J Prev Med 2016;50:398-401.

6 Vasan RS, Larson MG, Leip EP, et al. Assessment of frequency of progression to hypertension in non-hypertensive participants in the Framingham heart study: a cohort study. Lancet 2001;358:1682-6.

7 Usher-Smith JA, Silarova B, Schuit E, et al. Impact of provision of cardiovascular disease risk estimates to healthcare professionals and patients: a systematic review. BMJ Open 2015;5:e008717.

8 Lopez-Gonzalez AA, Aguilo A, Frontera M, et al. Effectiveness of the heart age tool for improving modifiable cardiovascular risk factors in a southern European population: a randomized trial. Eur J Prev Cardiol 2015;22:389-96.

9 Janssen KJM, Moons KGM, Kalkman CJ, et al. Updating methods improved the performance of a clinical prediction model in new patients. J Clin Epidemiol 2008;61:76-86.

10 Su T-L, Jaki T, Hickey GL, et al. A review of statistical updating methods for clinical prediction models. Stat Methods Med Res 2018;27:185-97.

11 Echouffo-Tcheugui JB, Batty GD, Kivimäki M, et al. Risk models to predict hypertension: a systematic review. PLoS One 2013;8:e67370.

12 Sun D, Liu J, Xiao L, et al. Recent development of risk-prediction models for incident hypertension: an updated systematic review. PLoS One 2017;12:e0187240.

13 Hamoen M, de Kroon MLA, Welten M, et al. Childhood prediction models for hypertension later in life: a systematic review. J Hypertens 2019;37:865-77.

14 Moher D, Shamseer L, Clarke M, et al. Preferred reporting items for systematic review and meta-analysis protocols (PRISMA-P) 2015 statement. Syst Rev 2015;4:1.
15 Munn Z, Stern C, Aromataris E, et al. What kind of systematic review should I conduct? A proposed typology and guidance for systematic reviewers in the medical and health sciences. BMC Med Res Methodol 2018;18:5.

16 Whelton PK, Carey RM, Aronow WS, et al. 2017 ACC/AHAVAAPA ABC/ACPM/AGS/APhA/ASH/ASPC/NMA/PCNA guideline for the prevention, detection, evaluation, and management of high blood pressure in adults: a report of the American College of Cardiology/ American heart association Task force on clinical practice guidelines. J Am Coll Cardiol 2018;71:e127-248.

17 Moher D, Liberati A, Tetzlaff J, et al. Preferred reporting items for systematic reviews and meta-analyses: the PRISMA statement. PLoS Med 2009;6:e1000097.

18 Kleinbaum DG, Dietz K, Gail M, et al. Logistic regression. New York: Springer-Verlag, 2002.

19 Riley RD, Moons KGM, Snell KIE, et al. A guide to systematic review and meta-analysis of prognostic factor studies. BMJ 2019;364:k4597.

20 Nieminen P, Lehtiniemi H, Vähäkangas K, et al. Standardised regression coefficient as an effect size index in summarising findings in epidemiological studies. Epidemiology, Biostatistics and Public Health 2013;10.

21 Fernández-Castilla B, Aloe AM, Declercq L, et al. Concealed correlations meta-analysis: a new method for synthesizing standardized regression coefficients. Behav Res Methods 2019;51:316-31.

22 Grace JB, Johnson DJ, Lefcheck JS, et al. Quantifying relative importance: computing standardized effects in models with binary outcomes. Ecosphere 2018;9:e02283.

23 Wolff RF, Moons KGM, Riley RD, et al. PROBAST: a tool to assess the risk of bias and applicability of prediction model studies. Ann Intern Med 2019;170:51-8.

24 Egger MDavey-Smith G, Altman D, eds. Systematic reviews in health care: meta-analysis in context. John Wiley \& Sons, 2008.

25 Hanley JA, McNeil BJ. The meaning and use of the area under a receiver operating characteristic $(\mathrm{ROC})$ curve. Radiology 1982;143:29-36.

26 Higgins JPT, Thompson SG, Deeks JJ, et al. Measuring inconsistency in meta-analyses. BMJ 2003;327:557-60.

27 Rothstein HR, Sutton AJ, Borenstein M. Publication bias in metaanalysis. In: Rothstein HR, Sutton AJ, Borenstein M, et al, eds. Publication bias in meta-analysis, 2005.

28 Egger M, Davey Smith G, Schneider M, et al. Bias in meta-analysis detected by a simple, graphical test. BMJ 1997;315:629-34.

29 Duval S, Tweedie R. Trim and fill: a simple funnel-plot-based method of testing and adjusting for publication bias in meta-analysis. Biometrics 2000;56:455-63.

30 Pearson TA, LaCroix AZ, Mead LA, et al. The prediction of midlife coronary heart disease and hypertension in young adults: the Johns Hopkins multiple risk equations. Am J Prev Med 1990;6:23-8.

31 Paynter NP, Cook NR, Everett BM, et al. Prediction of incident hypertension risk in women with currently normal blood pressure. $\mathrm{Am}$ J Med 2009;122:464-71.

32 Parikh NI, Pencina MJ, Wang TJ, et al. A risk score for predicting near-term incidence of hypertension: the Framingham heart study. Ann Intern Med 2008;148:102-10.

33 Debray TPA, Koffijberg H, Vergouwe Y, et al. Aggregating published prediction models with individual participant data: a comparison of different approaches. Stat Med 2012;31:2697-712.

34 Hemingway H, Riley RD, Altman DG. Ten steps towards improving prognosis research. BMJ 2009;339:b4184. 